Journal of Mathematics and Informatics

Vol. 20, 2021, 5-18

ISSN: 2349-0632 (P), 2349-0640 (online)

Published 8 February 2021

www.researchmathsci.org

DOI: http://dx.doi.org/10.22457/jmi.v20a02187

Journal of

Mathematics and Informatics

\title{
Research on the Operations of Independence Systems
}

Yonghong $\mathrm{Li}^{\mathrm{l}}$, Li $\mathrm{Li}^{2}$, Qingxi Shi ${ }^{3}$ and Huiming Duan ${ }^{4}$

\author{
${ }^{1,2,4}$ School of Science/Key Lab of Intelligent Analysis and Decision on Complex Systems \\ Chongqing University of Posts and Telecommunications, Chongqing 400065, P.R. China. \\ ${ }^{3}$ School of Computer Science and Information Engineering \\ Chongqing Technology and Business University, Chongqing 400067, P.R. China. \\ Corresponding author: liyh@cqupt.edu.cn
}

Received 1 December 2020; accepted 17 January 2021

Abstract. In this paper, the intersection and union of independence systems are considered in the same ground set. The sum and direct sum of independence systems are studied. Then the tree structure of independence systems is proposed and studied. Moreover, the intersection, union, sum and direct sum of independence systems are investigated in different ground sets.

Keywords: Independence systems; Intersection; Union; Sum; Tree structure

AMS Mathematics Subject Classification (2010): 52B40

\section{Introduction}

The independent set problem is widely studied in graph theory and it has important applications in many fields. There are mainly point-independent sets and edge-independent sets in graph theory, which often involve finding the optimal solution of the maximum independent set problem. The algorithms of finding the maximum independent set is often studied by scholars, such as the spanning tree algorithm. The graph coloring problem is a classical problem based on maximal independent set. The algorithm is to turn original problem into its sub-problem by obtaining a maximal independent set [1]. The maximum independent set problem with edge weights and its approximate algorithms are solved in [2]. The theory of maximal independent sets of graphs is applied to abstract the resources of education and a model is set up to achieve the function of auto-tabling in [3]. Recently, Chen [4] designed an exact algorithm based on branch and reduced to solve maximum independent set problem, which can get the exact solution of NP-hard problem in theory. And in [5], based on the graph theory, an independent set model suitable for ant colony algorithm is constructed. These problems are done on the basic of independent sets in graph theory and combinatorial mathematics.

In 1935, Whitney [6] first put forward the concept of matroids from the aspect of the independent set which provided a unifying abstract treatment of linear algebra and graph theory. Since then, the independent set axioms and other theory of matroids have been constructed and widely used. In [7], they are all done on the basic of independence systems of matroids, and then extended independent sets of matroids to independent sets of fuzzy matroids. In [8], many properties of fuzzy independence systems are found, such 
Yonghong Li, Li Li, Qingxi Shi and Huiming Duan

as the perfectness, regularity, refinement and so on.

As the core problem of NP-complete problem, the maximum independent set problem has been formed for a long time [9]. It is widely used in industrial process control, network design, large scale integrated circuit design and economic model analysis, such as time scheduling, register allocation, spectrum allocation, network communication, course scheduling, streaming media scheduling and so on in [10]. At the same time, it is also the problem of combinatorial optimization research in [11]. Many combinatorial structures [12] belong to the class of independence systems such as matroids, intersections of matroids and machings [13], which are worth studying. The independent structure appears in many branches of mathematics, such as linear independence in vector spaces, matchings in graphs and surfaces in projective geometries [14].

In this paper, the main goal is to study the properties of independence systems based on matroids and operations of sets. The concepts and properties of the sum and direct sum of independence systems on the same or different ground sets are introduced and studied. The operations and properties of independence systems are expanded to finite independence systems. This paper is organized as follows. In section 2, some basic notions and results related to independence systems are recalled. In section 3, the properties of operations of independence systems are studied. Conclusions is made in section 4.

\section{Preliminaries}

Firstly, the concept of independence system is introduced and the operations of sets are shown below.

Definition 2.1. $[6,8,24]$ Let $E$ be a nonempty finite set and $2^{E}$ be the set of all subsets of $E$, and $I \subseteq 2^{E}$. Then the pair $(E, I)$ is called a set system on a ground set $E$.

For the following conditions,

(i) $\varnothing \in I$.

(ii) (Hereditary property) If $A \in I$ and $B \subseteq A$, then $B \in I$.

(iii) (Exchange property) If $X, Y \in I$ and $|Y|>|X|$, then there exists an element $y \in Y \backslash X$ such that $X \cup\{y\} \in I$.

If $(E, I)$ satisfies conditions (i), (ii) and (iii), then $(E, I)$ is called a matroid.

If $(E, I)$ satisfies condition (ii), then $(E, I)$ is called an independence system.

For an independence system or a matroid $(E, I)$, the members of $I$ are called independent sets. $I$ is called a family of independent sets. A subset of $E$ that does not belong to $I$ is called a dependent set. The collection of dependent sets is denoted by $D$.

Since $E$ is finite, the number of all its subsets is finite. Therefore, the number of independence systems defined on a finite set $E$ is also finite.

Definition 2.2. [24] Let $X \subseteq E$ and $X \neq \varnothing . X$ is called a basis of an independence system $(E, I)$, if $X \in I$ and there does not exist a set $Y \in I$, such that $Y \supset X$.

That is, a maximal member of the family of independent sets $I$ is called a basis of $(E, I)$. The set of all bases is denoted by $B(I)$ or $B$. An empty independence system must have a basis at least, though all bases may not have the same cardinality. 
Research on the Operations of Independence Systems

Definition 2.3. [24] Let $(E, I)$ be an independence system and $A \subseteq E$. If the function $r: 2^{E} \rightarrow Z^{*}$ satisfies

$$
r(A)=\max \{\mid X \| X \subseteq A, X \in I\},
$$

where $Z^{*}$ is nonnegative integer. Then $r(A)$ is called the rank of set $A$ on $(E, I)$. Let $r(I)=\max \{\mid X \| X \subseteq B(I)\}$.

Then $r(I)$ is called the rank of an independence system $(E, I)$. That is, $r(I)$ is equal to the rank of a basis with the maximum rank of $(E, I) . X$ is called a maximum basis if the rank of $X$ is maximum among all the bases. When we discuss independence system $(E, I)$ later in this paper, we assume that $I \neq\{\varnothing\}$.

There are many properties of rank function of an independence system [17] [24].

Property 2.1. Suppose $r$ is the rank function of an independence system $(E, I)$ and nonempty sets $A, B \subseteq E, x, y \in E$ :

(i) $r(\varnothing)=0$.

(ii) $r(A) \leq|A|$, and if $A \in I$, then $r(A)=|A|(|A|$ is the cardinality of $A)$.

(iii) $r(A) \leq r(A \cup\{x\}) \leq r(A)+1$.

(iv) If $A \subseteq B$, then $r(A) \leq r(B)$.

(v) If $r(A \cup\{x\})=r(A \cup\{y\})=r(A)$, then $r(A \cup\{x\} \cup\{y\})=r(A)$.

The independence system $(E, I)$ is composed of subsets of a ground set $E$. Obviously, the elements of the independence system are sets. So some properties of independence system are related to the operation between sets, such as intersection, union, sum etc..

Definition 2.4. [25] Let $E$ be a ground set and $A, B$ and $C$ be subsets of $E$. Some operations are as follows:

(i) The intersection of $A$ and $B$ is denoted by $A \cap B=\{x \mid x \in A$ and $x \in B\}$.

(ii) The union of $A$ and $B$ is denoted by $A \cup B=\{x \mid x \in A$ or $x \in B\}$.

(iii) The complement of $A$ is denoted by $E \backslash A=\{x \mid x \in E$ and $x \notin A\}$.

(iv) The difference of $A$ and $B$ is denoted by $A-B=\{x \mid x \in A$ and $x \notin B\}$.

(v) The symmetric difference of $A$ and $B$ is denoted by $(A-B) \cup(B-A)$.

There are many properties among operations of sets, such as the associative law: $(A \cup B) \cup C=A \cup(B \cup C),(A \cap B) \cap C=A \cap(B \cap C)$.

The associative law will be applied to the families of independent sets, which makes the properties of two independence systems extend to finite ones in section 4 .

\section{The properties of operations for independence systems}

When discussing the properties of two independence systems according to the relationship 


\section{Yonghong Li, Li Li, Qingxi Shi and Huiming Duan}

between the two ground sets $E_{1}, E_{2}$, there are mainly two cases as follows:

(i) $E_{1}=E_{2}$, the intersection and union operations of two independence systems is actually on the same ground set.

(ii) $E_{1} \neq E_{2}$, the two ground sets are not equal and there exists the following cases:

(A) $E_{1} \cap E_{2}=\varnothing$, the two ground sets are separated from each other.

(B) $E_{1} \cap E_{2} \neq \varnothing$, two ground sets have at least one common element.

(a) $E_{1} \cap E_{2}=E$ and $0<|E| \leq \min \left\{\left|E_{1}\right|,\left|E_{2}\right|\right\}$, i.e. an element of the set $E_{1}$ does not belong to the set $E_{2}$ at least, that is ,there exists $x \in E_{1}$, and $x \notin E_{2}$.

(b) $E_{1} \subset E_{2}$ or $E_{2} \subset E_{1}$, that is, $E_{1}$ and $E_{2}$ have a proper inclusion relation.

Next, the operations and properties of the families of independent sets of the independence systems are studied based on the operations of the sets [25].

\subsection{On the same ground set}

In this section, two kinds of special independence systems are introduced, which are called the regular independence system and prefect independence system. And a tree structure about a special kind of independence systems is given, which is similar to the structure in [20], that is, there exists a tree structure of bases of independence systems on a same ground set. Then the operations of the families of independent sets of independence systems are studied according to the operations of sets. Finally, the properties of operations of independence systems on a same ground set are considered, which are similar to the results of matroids in [21].

Definition 3.1. Let $\left(E, I_{1}\right)$ and $\left(E, I_{2}\right)$ be the two distinct independence systems on the same ground set $E, I_{1}$ and $I_{2}$ be the two distinct families of independent sets, then $\left(E, I_{1}\right)$ is regular in $\left(E, I_{2}\right)$ if for each basis $B_{1} \in B\left(I_{1}\right)$, there always exists a basis $B_{2} \in B\left(I_{2}\right)$ such that $B_{1} \subseteq B_{2}$.

Definition 3.2. Let $(E, I)$ be an independence system on $E$ and $\{x\} \in I$ for some $x \in E$. Then $(E, I)$ is called a perfect independence system if any $X_{1}, X_{2} \in I$, then $X_{1} \cup X_{2} \in I$.

Then $I$ is called a perfect family of independent sets. If $(E, I)$ is a perfect independence system, there is only one basis. So, a perfect independence system and its basis are one-to-one correspondence relation.

Definition 3.3. Let $\left(E, I_{1}\right)$ and $\left(E, I_{2}\right)$ be two independence systems on $E$. If for any element $X \in I_{1}$, there exists at least an element $Y \in I_{2}$ such that $X \subseteq Y$, then $\left(E, I_{1}\right)$ is called a sub-independence system of $\left(E, I_{2}\right)$, denoted by $\left(E, I_{1}\right) \subseteq\left(E, I_{2}\right)$.

There exist finite independence systems on a finite set $E$, so there is an inclusion relationship between two or more independence systems on $E$. This inclusion relationship can be represented by a tree structure 
Example 3.1. Let $E=\{1,2,3,4\}$, there are four independence systems as follows:

$$
\begin{aligned}
& I_{1}=\{\varnothing,\{1\},\{2\}\}, I_{2}=\{\varnothing,\{1\},\{2\},\{3\},\{1,2\},\{2,3\},\{1,3\}\}, \\
& I_{3}=\{\varnothing,\{1\},\{2\},\{3\},\{1,2\},\{2,3\},\{1,3\},\{1,2,3\}\}, I_{4}=2^{E} .
\end{aligned}
$$

Obviously, $\left(E, I_{1}\right) \subseteq\left(E, I_{2}\right) \subseteq\left(E, I_{3}\right) \subseteq\left(E, I_{4}\right)$. It is easy to see that $\left(E, I_{1}\right)$ is regular in $\left(E, I_{2}\right)$ and $\left(E, I_{3}\right)$ and $\left(E, I_{4}\right),\left(E, I_{2}\right)$ is regular in $\left(E, I_{3}\right)$ and $\left(E, I_{4}\right),\left(E, I_{3}\right)$ is regular in $\left(E, I_{4}\right)$. There exists the transitivity property among them.

The perfect independence system is a special kind of matroids, which will be introduced later. In Example 3.1, $\left(E, I_{3}\right)$ and $\left(E, I_{4}\right)$ are perfect independence systems and also matroids. But $\left(E, I_{1}\right)$ and $\left(E, I_{2}\right)$ are only independence systems.

It is known that there is a inclusion relationship of the sequence of these families of independent sets, that is, $I_{4} \supset I_{3} \supset I_{2} \supset I_{1}$. The families of independent sets $I_{1}, I_{2}$ and $I_{3}$ are regular in $I_{4}$. The bases of $I_{4}, I_{3}, I_{2}$ and $I_{1}$ can form a tree structure, see Fig. 1.

From the tree structure, the bases of the independence systems have an inclusion relationship among the families of independent sets. All bases of the minimum independence system are leaves of the tree paper, the tree structure of the inclusion relation of the families of independent sets among independence systems is discussed and some properties are studied.

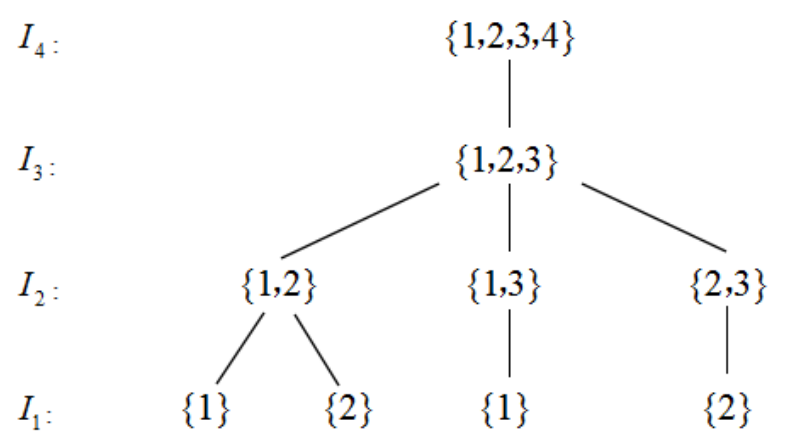

Figure 1: The tree structure of independence systems

From the definition of a perfect independence system, it is easy to obtain the following properties.

Property 3.1. The hereditary property holds in the family of independent sets for an independence system.

Property 3.2. Let $(E, I)$ be a perfect independence system. Then $X_{1} \cup X_{2} \in I$ for any $X_{1}, X_{2} \in I$. 
Example 3.2. Let $E=\{1,2,3\}$. Let $I_{1}=\{\varnothing,\{1\},\{2\}\}, I_{2}=\{\varnothing,\{1\},\{2\},\{1,2\}\}$.

Obviously, $\{1\} \cup\{2\} \notin I_{1},\{1\} \cup\{2\} \in I_{2}$. Then $\left(E, I_{2}\right)$ is not a perfect independence system, but $\left(E, I_{2}\right)$ is a perfect independence system.

Property 3.3. A perfect independence system is a special kind of crisp matroid.

Proof: Let $(E, I)$ be a perfect independence system. Then the conditions (i)(ii) of Definition 2.1 hold in $(E, I)$. We only show that condition (iii) of Definition 2.1 holds in $(E, I)$.

Suppose that $X_{1}, X_{2} \in I$ and $\left|X_{2}\right|>\left|X_{1}\right|$. By the hypothesis, for any $x \in X_{2} \backslash X_{1}$, we have $X_{1} \cup\{x\} \subseteq X_{1} \cup X_{2} \in I$, it implies that $X_{1} \cup\{x\} \in I$. Hence, $(E, I)$ is a crisp matroid.

Therefore, a perfect independence system is a special kind of crisp matroid.

For example, the perfect independence system $\left(E, 2^{E}\right)$ is a matroid, but not vice versa.

According to the number of perfect independence systems on a finite set and the property of their bases, there also exists a tree structure about bases of the special kind of matroids.

Example 3.3. For $E_{1}=\{1,2\}$, there exist three perfect independence systems:

$$
I_{11}=\{\varnothing,\{1\}\}, \quad I_{12}=\{\varnothing,\{2\}\}, \quad I_{13}=\{\varnothing,\{1\},\{2\},\{1,2\}\} .
$$

For $E_{2}=\{1,2,3\}$, there exist seven perfect independence systems:

$$
\begin{gathered}
I_{21}=\{\varnothing,\{1\}\}, \quad I_{22}=\{\varnothing,\{2\}\}, \quad I_{23}=\{\varnothing,\{3\}\}, \quad I_{24}=\{\varnothing,\{1\},\{2\},\{1,2\}\}, \\
I_{25}=\{\varnothing,\{1\},\{3\},\{1,3\}\}, \quad I_{26}=\{\varnothing,\{2\},\{3\},\{2,3\}\}, \\
I_{27}=\{\varnothing,\{1\},\{2\},\{3\},\{1,2\},\{2,3\},\{1,3\},\{1,2,3\}\} .
\end{gathered}
$$

Then their tree structures can be constructed as Fig.2.

The tree structure of three perfect independence systems on $E_{1}$ is on left in Fig.2 and the tree structure of seven perfect independence systems on $E_{2}$ is on the right in Fig.2. Every element of the tree structures is a basis of a perfect independence system. Each element and its subsets form a perfect independence system in the tree structure. 
Research on the Operations of Independence Systems
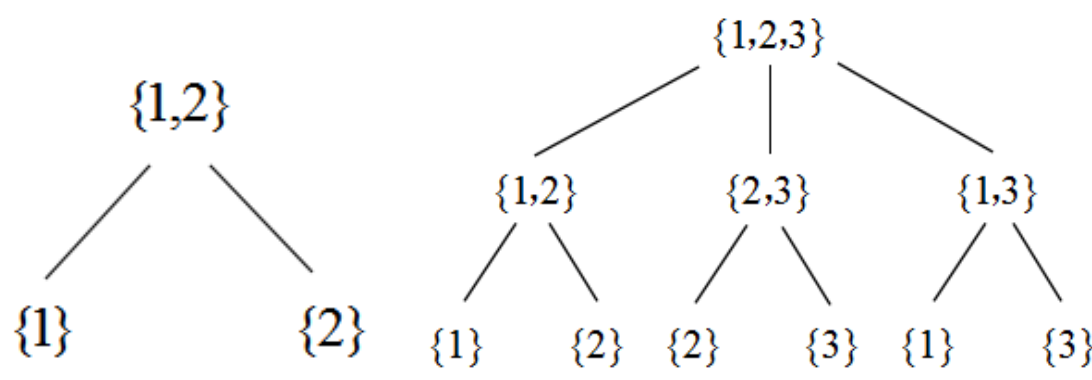

Figure 2: The tree structures of perfect independence systems

Property 3.4. Let $E$ be a finite set with $|E|=n$ and $\mathrm{I}$ be the set of all families of independent sets on $E$. Then, when $n \geq 1$, the number of perfect independence systems on $E$ is $2^{n}-1$, i.e., $|\mathrm{I}|=2^{n}-1$.

Property 3.5. If $(E, I)$ is a perfect independence system, then it has only one basis.

Proof: Assume that $B_{1}$ and $B_{2}$ are bases of a perfect independence system $(E, I)$, then $B=B_{1} \cup B_{2} \in I$. It is contradiction with $B_{1}$ and $B_{2}$ which are bases. Hence, there is only one basis for a perfect independence system.

The following theorem and its corollary studied in [26] are introduced.

Theorem 3.1. Suppose that $\left(E, I_{1}\right)$ and $\left(E, I_{2}\right)$ are the two different independence systems on $E$, then $\left(E, I_{1} \cap I_{2}\right)$ and $\left(E, I_{1} \cup I_{2}\right)$ are also independence systems on $E$

Corollary 3.1. Let $\left(E, I_{i}\right)(i=1,2, \cdots, n)$ be different independence systems on a finite set $E$, then $\left(E, \bigcup_{i=1}^{n} I_{i}\right)$ and $\left(E, \bigcap_{i=1}^{n} I_{i}\right)$ are also independence systems on $E$. That is, the union or intersection of finite families of independent sets on $E$ are also families of independent sets.

Next, the properties of the sum and direct sum of finite independence systems are studied, which are similar to matroids [22] and [23]. Firstly, we introduced Theorem 3.2 which is studied in [26].

Theorem 3.2. Let $\left(E, I_{1}\right)$ and $\left(E, I_{2}\right)$ be two independence systems on $E$ and let $I_{1}+I_{2}=\left\{A \cup B \mid A \in I_{1}, B \in I_{2}\right\}$.

Then $\left(E, I_{1}+I_{2}\right)$ is an independence system.

Definition 3.4. $\left(E, I_{1}+I_{2}\right)$ defined in Theorem 3.2 is called the sum of $\left(E, I_{1}\right)$ and $\left(E, I_{2}\right)$. If $I_{1} \cap I_{2}=\{\varnothing\}$, then the sum of $\left(E, I_{1}\right)$ and $\left(E, I_{2}\right)$ is called the direct sum, 


\section{Yonghong Li, Li Li, Qingxi Shi and Huiming Duan}

denoted by $\left(E, I_{1}\right) \oplus\left(E, I_{2}\right)=\left(E, I_{1} \oplus I_{2}\right)$.

Now, we study the properties of the sum of $\left(E, I_{1}\right)$ and $\left(E, I_{2}\right)$ below.

Theorem 3.3. Let $\left(E, I_{1}\right)$ and $\left(E, I_{2}\right)$ be two independence systems on $E$, suppose $\left(E, I_{1}+I_{2}\right)$ is the sum of $\left(E, I_{1}\right)$ and $\left(E, I_{2}\right)$. Then $r_{1}\left(I_{1}\right) \leq r\left(I_{1}+I_{2}\right)$ and $r_{2}\left(I_{2}\right) \leq r\left(I_{1}+I_{2}\right)$, where $r, r_{1}, r_{2}$ are the rank functions of $\left(E, I_{1}+I_{2}\right),\left(E, I_{1}\right),\left(E, I_{2}\right)$ respectively.

Proof: Let $A$ be a basis of $\left(E, I_{1}\right)$ with maximum rank $r_{1}\left(I_{1}\right)$. Suppose $\left(E, I_{1}+I_{2}\right)$ is the sum of $\left(E, I_{1}\right)$ and $\left(E, I_{2}\right)$. Obviously, $A=A \cup \varnothing \in I_{1}+I_{2}$. Hence, There exists a basis $B$ of $\left(E, I_{1}+I_{2}\right)$ such that $A \subseteq B$. It implies that $|A| \leq|B| \leq r\left(I_{1}+I_{2}\right)$. Note that $|A|=r_{1}\left(I_{1}\right)$. Then $r_{1}\left(I_{1}\right) \leq r\left(I_{1}+I_{2}\right)$.

Similarly, $r_{2}\left(I_{2}\right) \leq r\left(I_{1}+I_{2}\right)$.

Theorem 3.4. Let $\left(E, I_{1}+I_{2}\right)$ be the sum of two independence system $\left(E, I_{1}\right)$ and $\left(E, I_{2}\right)$. Suppose that $r_{1}\left(I_{1}\right) \leq r_{2}\left(I_{2}\right)=r\left(I_{1}+I_{2}\right)$, where $r, r_{1}, r_{2}$ are the rank functions of $\left(E, I_{1}+I_{2}\right),\left(E, I_{1}\right),\left(E, I_{2}\right)$, respectively. Then

(i) If $A$ is a basis of $\left(E, I_{1}\right)$ with maximum rank $r_{1}\left(I_{1}\right)$, then there exists a basis $B$ of $\left(E, I_{2}\right)$ with maximum rank $r_{2}\left(I_{2}\right)$ such that $A \subseteq B$.

(ii) $\left(E, I_{2}\right)=\left(E, I_{1}+I_{2}\right)$.

Proof. (i) Assume that for any basis $B$ of $\left(E, I_{2}\right)$ with maximum rank $r_{2}\left(I_{2}\right), A \subseteq B$ does't hold. Then there exists $x \in A$ such that $x \notin B$. It follows that $|A \cup B|>|B|=r_{2}\left(I_{2}\right)$. Moreover, $A \cup B \in I_{1}+I_{2}$. Then $|A \cup B| \leq r\left(I_{1}+I_{2}\right)$. Thus, $r\left(I_{1}+I_{2}\right)>r_{2}\left(I_{2}\right)$. This is contradiction with $r_{2}\left(I_{2}\right)=r\left(I_{1}+I_{2}\right)$.

(ii) Obviously, $I_{2} \subseteq I_{1}+I_{2}$.

On the contrary, for any $A \in I_{1}+I_{2}$, there exist $B \in I_{1}, N \in I_{2}$ such that $A=B \cup N$. Then there exists a basis $C \in I_{1}$ with maximum rank $r_{1}\left(I_{1}\right)$ such that $B \subseteq C$. From (i), there exists a basis $M \in I_{2}$ with maximum rank $r_{2}\left(I_{2}\right)$ such that $C \subseteq M$. It follows that $B \subseteq M$. It implies that $A=B \cup N \subseteq M \cup N \in I_{2}$. Then $A \in I_{2}$. Thus $I_{1}+I_{2} \subseteq I_{2}$.

Therefore, $I_{2}=I_{1}+I_{2}$, i.e., $\left(E, I_{2}\right)=\left(E, I_{1}+I_{2}\right)$.

Theorem 3.5. Let $\left(E, I_{1}+I_{2}\right)$ be the sum of two independence systems $\left(E, I_{1}\right)$ and $\left(E, I_{2}\right)$. Then any basis of $\left(E, I_{1}+I_{2}\right)$ can be denoted by $M \cup N$, where $M$ is a basis of $\left(E, I_{1}\right)$ and $N$ is a basis of $\left(E, I_{2}\right)$.

Proof: Let $A$ be a basis of $\left(E, I_{1}+I_{2}\right)$, then $A=A_{1} \cup A_{2}$ and $A_{1} \in I_{1}, A_{2} \in I_{2}$. It 


\section{Research on the Operations of Independence Systems}

implies that there exist respective bases $M$ and $N$ of $I_{1}$ and $I_{2}$, such that $A_{1} \subseteq M$ and $A_{2} \subseteq N$. It follows that $A \subseteq M \cup N \in I_{1}+I_{2}$. Note that $A$ be a basis of $\left(E, I_{1}+I_{2}\right)$, Hence, $A=M \cup N$.

Note that the upper Theorems are similar to the results in matroid theory. It is easy to obtain the following property according to Theorem 3.5.

Corollary 3.2. Let $\left(E, I_{1} \oplus I_{2}\right)$ be the direct sum of two independence system $\left(E, I_{1}\right)$ and $\left(E, I_{2}\right)$. Suppose that $M$ is a basis of $\left(E, I_{1} \oplus I_{2}\right)$. Then $M$ can be denoted by $N \cup(M \backslash N)$, where $N$ is a basis of $\left(E, I_{1}\right)$ and $M \backslash N$ is a basis of $\left(E, I_{2}\right)$.

Remark 3.1. The difference of the families of independence systems may not be an independence system. Suppose $\left(E, I_{1}\right)$ and $\left(E, I_{2}\right)$ are the two different independence systems on $E$. If $I_{1} \cap I_{2}=\{\varnothing\}$, then $I_{2}-I_{1}=I_{2}-\{\varnothing\}, I_{1}-I_{2}=I_{1}-\{\varnothing\}$, then $\left(E,\left(I_{1}-I_{2}\right) \cup\{\varnothing\}\right)=\left(E, I_{1}\right)$ and $\left(E,\left(I_{2}-I_{1}\right) \cup\{\varnothing\}\right)=\left(E, I_{2}\right)$ are independence systems.

But when $I_{1} \cap I_{2} \neq\{\varnothing\}$, the results don't hold. For example, $E=\{1,2,3\}$, $I_{1}=\{\varnothing,\{1\}\} \quad I_{2}=\{\varnothing,\{1\},\{2\}\} \quad I_{3}=\{\varnothing,\{1\},\{2\},\{1,2\}\} \quad$ and $I_{4}=\{\varnothing,\{1\},\{3\},\{1,3\}\} \quad, \quad I_{2}-I_{1}=\{\{2\}\} \quad, \quad I_{3}-I_{2}=\{\{1,2\}\} \quad$ and $I_{4}-I_{3}=\{\{3\},\{1,3\}\}$. Then $\left(E,\left(I_{2}-I_{1}\right) \cup\{\varnothing\}\right)$ is an independence system, $\left(E,\left(I_{3}-I_{2}\right) \cup\{\varnothing\}\right)$ and $\left(E,\left(I_{4}-I_{3}\right) \cup\{\varnothing\}\right)$ are not independence systems.

\subsection{On the different ground sets}

In this section, we will discuss the intersection, union, sum and direct sum of independent systems when the ground sets are different.

Theorem 3.6. Let $\left(E_{1}, I_{1}\right)$ and $\left(E_{2}, I_{2}\right)$ be two independence systems, then $\left(E_{1} \cup E_{2}, I_{1} \cup I_{2}\right)$ is still an independence system.

Proof: Let $\left(E_{1}, I_{1}\right),\left(E_{2}, I_{2}\right)$ be two independence systems, then the two families of independent sets $I_{1}, I_{2}$ satisfy the hereditary property on the respective ground set $E_{1}, E_{2}$ , respectively. Now we prove the set $I_{1} \cup I_{2}$ is a family of independent sets. Let $A \in I_{1} \cup I_{2}$, then $A \in I_{1}$ or $A \in I_{2}$. Let $A \in I_{1}$. Because $I_{1}$ is a family of independent sets, from the hereditary property of independence system, for any $B \subseteq A$, we have $B \in I_{1}$. It follows that $B \in I_{1} \cup I_{2}$. Hence, $I_{1} \cup I_{2}$ is an independent set. Therefore, the set system $\left(E_{1} \cup E_{2}, I_{1} \cup I_{2}\right)$ is an independence system.

From the above property, the following corollary can be obtained.

Corollary 3.3. If $\left(E_{i}, I_{i}\right)(i=1,2, \cdots, n)$ are independence systems on $E_{1}, E_{2}, \cdots, E_{n}$, 
Yonghong Li, Li Li, Qingxi Shi and Huiming Duan

respectively. Then $\left(\bigcup_{i=1}^{n} E_{i}, \bigcup_{i=1}^{n} I_{i}\right)$ is an independence system.

Next, we discuss the sum of independence systems on the different ground set.

Theorem 3.7. Suppose that $\left(E_{1}, I_{1}\right)$ and $\left(E_{2}, I_{2}\right)$ are two independence systems. Let

$$
I_{1}+I_{2}=\left\{A \cup B \mid A \in I_{1}, B \in I_{2}\right\} .
$$

Then $\left(E_{1} \cup E_{2}, I_{1}+I_{2}\right)$ is an independence system.

Proof: Let $A \in I_{1}+I_{2}$ and $B \subseteq A$. Then there exist $A_{1} \in I_{1}$ and $A_{2} \in I_{2}$ such that $A=A_{1} \cup A_{2}$. It follows that $B \subseteq A_{1} \cup A_{2}$. Let $B_{1}=B \backslash A_{2}$ and $B_{2}=B \backslash A_{1}$, then $B_{1} \subseteq A_{1}$ and $B_{2} \subseteq A_{2}$ and $B=B_{1} \cup B_{2}$. By the hypothesis and the definition of independence system, $B_{1} \in I_{1}$ and $B_{2} \in I_{2}$. Then $B_{1} \cup B_{2} \in I_{1}+I_{2}$, i.e., $B \in I_{1}+I_{2}$. Therefore, $\left(E_{1} \cup E_{2}, I_{1}+I_{2}\right)$ is an independence system.

From Theorem 3.7, the sum of two independence systems is defined and the its properties are studied.

Definition 3.6. Suppose $I_{1}+I_{2}$ is defined in Theorem 3.7. Then $\left(E_{1} \cup E_{2}, I_{1}+I_{2}\right)$ is called the sum of $\left(E_{1}, I_{1}\right)$ and $\left(E_{2}, I_{2}\right)$, denoted by

$$
\left(E_{1}, I_{1}\right)+\left(E_{2}, I_{2}\right)=\left(E_{1} \cup E_{2}, I_{1}+I_{2}\right) \text {. If } E_{1} \cap E_{2}=\varnothing \text {, then }\left(E_{1} \cup E_{2}, I_{1}+I_{2}\right)
$$

is called the direct sum of $\left(E_{1}, I_{1}\right)$ and $\left(E_{2}, I_{2}\right)$, denoted by

$$
\left(E_{1}, I_{1}\right) \oplus\left(E_{2}, I_{2}\right)=\left(E_{1} \cup E_{2}, I_{1} \oplus I_{2}\right) \text {. }
$$

It is easy to see that $I_{1} \subseteq I_{1}+I_{2}$ and $I_{2} \subseteq I_{1}+I_{2}$. When two families of independent sets $I_{1}, I_{2}$ are given, the family of independent sets $I$ of the direct sum is determined. Next, the properties of bases and ranks of the sum are introduced. Note that for the different ground sets $E_{1}$ and $E_{2}, r\left(I_{1}\right)$ should be treated in the union $E_{1} \cup E_{2}$.

Theorem 3.8. Let $\left(E_{1}, I_{1}\right)$ and $\left(E_{2}, I_{2}\right)$ be two independence systems on $E_{1}$ and $E_{2}$, respectively. Suppose that $\left(E_{1} \cup E_{2}, I_{1}+I_{2}\right)$ is the sum of them. Then $r_{1}\left(I_{1}\right) \leq r\left(I_{1}+I_{2}\right)$ and $r_{2}\left(I_{2}\right) \leq r\left(I_{1}+I_{2}\right)$, where $r, r_{1}, r_{2}$ are the rank functions of $\left(E_{1} \cup E_{2}, I_{1}+I_{2}\right),\left(E_{1}, I_{1}\right),\left(E_{2}, I_{2}\right)$, respectively.

Proof: Let $A$ be a basis of $\left(E, I_{1}\right)$ with maximum rank $r_{1}\left(I_{1}\right)$. Suppose that $\left(E_{1} \cup E_{2}, I_{1}+I_{2}\right)$ is the sum of $\left(E, I_{1}\right)$ and $\left(E, I_{2}\right)$. Obviously, $A=A \cup \varnothing \in I_{1}+I_{2}$ . Hence, There exists a basis $B$ of $\left(E_{1} \cup E_{2}, I_{1}+I_{2}\right)$ such that $A \subseteq B$. It implies that $|A| \leq|B| \leq r\left(I_{1}+I_{2}\right)$. Note that $|A|=r_{1}\left(I_{1}\right)$. Then $r_{1}\left(I_{1}\right) \leq r\left(I_{1}+I_{2}\right)$.

Similarly, $r_{2}\left(I_{2}\right) \leq r\left(I_{1}+I_{2}\right)$. 
Research on the Operations of Independence Systems

Theorem 3.9. Let $\left(E_{1} \cup E_{2}, I_{1}+I_{2}\right)$ be the sum of the two independence system $\left(E_{1}, I_{1}\right)$ and $\left(E_{2}, I_{2}\right)$. Then any basis of $\left(E_{1} \cup E_{2}, I_{1}+I_{2}\right)$ can be denoted by $M \cup N$ , and $M$ is a basis of $\left(E_{1}, I_{1}\right), N$ is a basis of $\left(E_{2}, I_{2}\right)$.

Proof: Let $A$ be a basis of $\left(E_{1} \cup E_{2}, I_{1}+I_{2}\right)$, then $A=A_{1} \cup A_{2}$ and $A_{1} \in I_{1}, A_{2} \in I_{2}$ . It implies that there exist respective bases $M$ and $N$ of $I_{1}$ and $I_{2}$, such that $A_{1} \subseteq M$ and $A_{2} \subseteq N$. It follows that $A \subseteq M \cup N \in I_{1}+I_{2}$. Note that $A$ be a basis of $\left(E, I_{1}+I_{2}\right)$, Hence, $A=M \cup N$.

From Theorem 3.9, the following property can be obtained.

Property 3.6. Let $\left(E_{1} \cup E_{2}, I_{1} \oplus I_{2}\right)$ be the direct sum of the two independence system $\left(E_{1}, I_{1}\right)$ and $\left(E_{2}, I_{2}\right)$. Suppose that $M$ is a basis of $\left(E_{1} \cup E_{2}, I_{1} \oplus I_{2}\right)$. Then $M$ can be denoted by $N \cup(M \backslash N)$ and $N$ is a basis of $\left(E_{1}, I_{1}\right), M \backslash N$ is a basis of $\left(E_{2}, I_{2}\right)$.

Theorem 3.10. Let $\left(E_{1} \cup E_{2}, I_{1}+I_{2}\right)$ be the sum of the two independence system $\left(E_{1}, I_{1}\right)$ and $\left(E_{2}, I_{2}\right)$ and $A \subseteq E_{1} \cup E_{2}$. Then $A$ is an independent set of $\left(E_{1} \cup E_{2}, I_{1}+I_{2}\right)$ if and only if $A \cap E_{1}$ and $A \cap E_{2}$ are independent sets of $\left(E_{1}, I_{1}\right)$ and $\left(E_{2}, I_{2}\right)$ respectively.

Proof: (Necessity) Since $A \subseteq E_{1} \cup E_{2}$ is an independent set of $\left(E_{1} \cup E_{2}, I_{1}+I_{2}\right)$, then $A \in I_{1}+I_{2}$. It follows that there exist $A_{1} \in I_{1}, A_{2} \in I_{2}$ such that $A=A_{1} \cup A_{2}$. Then $A_{1}, A_{2}$ are the independent sets of $\left(E_{1}, I_{1}\right),\left(E_{2}, I_{2}\right)$, respectively. Obviously, $A \cap E_{1}=A_{1}, A \cap E_{2}=A_{2}$ are the independent sets of $\left(E_{1}, I_{1}\right),\left(E_{2}, I_{2}\right)$, respectively.

(Sufficiency) Suppose that $A \subseteq E_{1} \cup E_{2}$ and $A \cap E_{1}$ and $A \cap E_{2}$ are independent sets of $\left(E_{1}, I_{1}\right)$ and $\left(E_{2}, I_{2}\right)$, respectively. Then $A \cap E_{1} \in I_{1}$ and $A \cap E_{2} \in I_{2} \quad$. It follows that $\left(A \cap E_{1}\right) \cup\left(A \cap E_{2}\right) \in I_{1}+I_{2}$. Note that $\left(A \cap E_{1}\right) \cup\left(A \cap E_{2}\right)=A \cap\left(E_{1} \cup E_{2}\right)=A$. Then $A \in I_{1}+I_{2}$. That is, $A$ is an independent set of $\left(E_{1} \cup E_{2}, I_{1}+I_{2}\right)$.

From Theorem 3.10, it is easy to get the following property.

Property 3.7. Let $\left(E_{1} \cup E_{2}, I_{1}+I_{2}\right)$ be the sum of the two independence system $\left(E_{1}, I_{1}\right)$ and $\left(E_{2}, I_{2}\right)$. Suppose $r, r_{1}, r_{2}$ are the rank functions of $\left(E_{1} \cup E_{2}, I_{1}+I_{2}\right),\left(E_{1}, I_{1}\right),\left(E_{2}, I_{2}\right)$, respectively. Then for any $A \subseteq E_{1} \cup E_{2}$, $r(A) \leq r_{1}\left(A \cap E_{1}\right)+r_{2}\left(A \cap E_{2}\right)$.

Proof: For any $A \subseteq E_{1} \cup E_{2}$, let $B$ be the maximal independent set contained by $A$, 
Yonghong Li, Li Li, Qingxi Shi and Huiming Duan

i.e., $B \in I_{1}+I_{2}$ and $r(A)=r(B)=|B|$. Then from Theorem 3.10, $B \cap E_{1} \in I_{1}$ and $B \cap E_{2} \in I_{2}$. It follows that $r_{1}\left(B \cap E_{1}\right)=\left|B \cap E_{1}\right|$ and $r_{2}\left(B \cap E_{2}\right)=\left|B \cap E_{2}\right|$. Note that $\left|B \cap E_{1}\right|+\left|B \cap E_{2}\right| \geq\left|\left(B \cap E_{1}\right) \cup\left(B \cap E_{2}\right)\right|=\left|B \cap\left(E_{1} \cup E_{2}\right)\right|=|B|$. Therefore, $r(A) \leq r_{1}\left(A \cap E_{1}\right)+r_{2}\left(A \cap E_{2}\right)$.

From Property 3.7, we can obtain the following corollary.

Corollary 3.4. Let $\left(E_{1} \cup E_{2}, I_{1} \oplus I_{2}\right)$ be the direct sum of the two independence system $\left(E_{1}, I_{1}\right)$ and $\left(E_{2}, I_{2}\right)$. Suppose $r, r_{1}, r_{2}$ are the rank functions of $\left(E_{1} \cup E_{2}, I_{1} \oplus I_{2}\right),\left(E_{1}, I_{1}\right),\left(E_{2}, I_{2}\right)$, respectively. Then for any $A \subseteq E_{1} \cup E_{2}$, $r(A)=r_{1}\left(A \cap E_{1}\right)+r_{2}\left(A \cap E_{2}\right)$.

The previous properties is about the union of independence systems. Next, we discuss the intersection of independence systems.

Theorem 3.11. Let $\left(E_{1}, I_{1}\right)$ and $\left(E_{2}, I_{2}\right)$ be two independence systems. Then $\left(E_{1} \cup E_{2}, I_{1} \cap I_{2}\right)$ is still an independence system.

Proof: Obviously, $I_{1} \cap I_{2}$ is the family of subsets of $E_{1} \cup E_{2}$. Let $A \in I_{1} \cap I_{2}$ and $B \subseteq A$. Then $A \in I_{1}$ and $A \in I_{2}$. From the definition of independence system, we have that $B \in I_{1}$ and $B \in I_{2}$. It follows that $B \in I_{1} \cap I_{2}$.

Therefore, $\left(E_{1} \cup E_{2}, I_{1} \cap I_{2}\right)$ is still an independence system.

The following corollary can be obtained from Theorem 3.11.

Corollary 3.5. If $\left(E_{i}, I_{i}\right)(i=1,2, \cdots, n)$ are independence systems on $E_{1}, E_{2}, \cdots, E_{n}$, respectively. Then the intersection of the independence systems $\left(\bigcup_{i=1}^{n} E_{i}, \bigcap_{i=1}^{n} I_{i}\right)$ is still an independence system.

\section{Conclusion}

Independent structure plays an important role in many mathematical branches. The properties of independence systems are of great significance for matroid structure and many properties of matroid can be extended to independence systems. In this paper, some operations such as intersection, union, sum and direct sum of independence systems are considered. The tree structure of independence systems is proposed and studied. Next, we will continue to study the structure and similarities between the independence system and the topology. The properties of the independence systems can applies to topology.

\section{REFERENCES}

1. D.T.Hoang and N.T.Tran, Coverings, matchings and the number of maximal independent sets of graph, Australasian Journal of Combinatorics, 73(3) (2019) 424- 
Research on the Operations of Independence Systems

431.

2. H.Zhang and H.Zhu, Edge weighted maximum independent set problem and an approximate algorithm for i, Computer science, 31(9) (2004) 140.

3. Q.F.Li, Application of the maximal independent sets in college time-table system, Journal of Guangxi Academy of Sciences, 22(4) (2006) 339-341.

4. J.Z.Chen, A.B.Ning and Z.B.Zhi, Exact algorithm for maximum independent set problem in graph theory, Computer Engineering and Applications, 52(1) (2016) 2022.

5. W.J.Chai and C.Zhang, Double ant colony algorithm of multi-atellite mission planning based on graph theory, Radio Engineering, 49(6) (2019) 534-539.

6. H.Whitney, On the abstract properties of linear dependence, American Journal of Mathematics, 57(3) (1935) 509-533.

7. X.N.Li, S.Y.Liu and H.J.Yi, The independent set axioms, basis axioms and cyclic axioms of hypermatroids, Chinese Science: Mathematics, 46(9) (2016) 1351.

8. L.A.Novak, On fuzzy independence set systems, Fuzzy Sets and Systems, 91(3) (1997) 365-374.

9. Z.Z.Chen, Approximation algorithms for independent sets in map graphs, Journal of Algorithms, 41(1) (2001) 20-40.

10. E.Davies, M.Jenssen and W.Perkins, On the average size of independent sets in triangle-free graphs, Proceedings of the American Mathematical Society, 146(1) (2017) 111-124.

11. D.J.A.Welsh, V.Bryant and H.Perfect, Independence theory in combinatorics, Mathematical Gazette, 65(433) (1980) 228.

12. V.E.Levit and E.Mandrescu, On the structure of the minimum critical independent set of a graph, Discrete Mathematics, 313(5) (2013) 605-610.

13. R.Balakrishnan and K.Ranganathan, Independent sets and matchings, A Textbook of Graph Theory, 10(5) (2012) 7-115.

14. X.N.Li and H.J.Yi, Fuzzy bases of fuzzy independence set systems, Fuzzy Sets and Systems, 311(1) (2016) 99-111.

15. G.J.Roy and W.Voxman, Bases of fuzzy matroids, Fuzzy Sets and Systems, 31(2) (1989) 253-261.

16. R.G.Jr and W.Voxman, Fuzzy circuits, Fuzzy Sets and Systems, 32(1) (1989) 35-43.

17. X.Xiu and F.G.Shi, Rank functions for closed and perfect [0,1]-matroids, Hacettepe Journal of Mathematics and Statistics, 39 (2010) 31-39.

18. R.S.Yang, The incidence matrix of base, Journal of Chongqing Normal University (Natural Science Edition), 18(4) (2001) 67-70.

19. X.Xiu and F.G.Shi, M-fuzzying bases, Proyecciones Journal of Mathematic, 28(3) (2009) 271-283.

20. H.F.Harary, On the tree graph of a matroid, SIAM Journal on Applied Mathematics, 22(2) (1972) 187-193.

21. C.D.Li and D.Y.Wu, Duality and hyperplanes of fuzzy matroids, Journal of Chongqing University (Natural Science Edition), 25(4) (2002) 116-119.

22. J.E.Bonin and J.P.S.Kung, Semidirect Sums of Matroids, Annals of Combinatorics, 19(1) (2015) 7-27.

23. H.F.Xiang, D.Y.Wu and C.D.Li, Spanning properties of fuzzy matroids, Journal of Chongqing University (Natural Science Edition), 28(5) (2005) 102-105. 
Yonghong Li, Li Li, Qingxi Shi and Huiming Duan

24. L.Matthews, Closure in Independence Systems, Mathematics of Operations Research, 7(2) (1982) 159-171.

25. D.J.Qi, Independent of four operations of set, Natural Sciences Journal of Harbin Normal University, 21(13) (2005) 23-24.

26. B.Korte and J.Vygen, Combinatorial optimization: theory and algorithms, 21(2012) 321-374. 